\title{
Indirect dark matter search with the balloon-borne PEBS detector
}

\section{Henning Gast*, Roman Greim, Thomas Kirn, Gregorio Roper Yearwood, and Stefan Schael}

I. Physikalisches Institut B, RWTH Aachen, Aachen, Germany

E-mail: henning@physik.rwth-aachen.de

greimephysik.rwth-aachen.de

kirn@physik.rwth-aachen.de

roper@physik.rwth-aachen.de

schaelaphysik.rwth-aachen.de

\begin{abstract}
A precision measurement of the cosmic-ray positron spectrum may help to solve the puzzle of the nature of dark matter. Pairwise annihilation of neutralinos, predicted by some supersymmetric extensions to the standard model of particle physics, may leave a distinct feature in the cosmicray positron spectrum.

As the available data are limited both in terms of statistics and energy range, we are developing a balloon-borne detector (PEBS) with a large acceptance of $4000 \mathrm{~cm}^{2} \mathrm{sr}$. A superconducting magnet creating a field of $0.8 \mathrm{~T}$ and a tracking device consisting of scintillating fibers of $250 \mu \mathrm{m}$ diameter with silicon photomultiplier readout will allow rigidity and charge determination to energies above $100 \mathrm{GeV}$. The dominant proton background is suppressed by the combination of an electromagnetic calorimeter and a transition radiation detector consisting of fleece layers interspersed with straw-tube proportional counters. The calorimeter uses a sandwich of tungsten and scintillating fibers that are again read out by silicon photomultipliers.

The design study, based on a detailed Geant4 simulation and testbeam measurements, will be presented along with an interpretation of the currently available positron data in the context of the mSUGRA model. The constraints that future precise measurements could put on this model will be discussed.
\end{abstract}

Identification of dark matter 2008

August 18-22, 2008

Stockholm, Sweden

* Speaker. 


\section{Detector design}

As an experiment designed to measure the positron component in the cosmic rays with high precision, the Positron-Electron-Balloon-Spectrometer (PEBS) has to meet several crucial requirements: First of all, the geometric acceptance needs to be large due to the small flux of positrons. A clean positron sample can only be obtained if a suppression of the predominant proton background on the level of one in one million is achieved. In addition, a good momentum resolution is necessary for charge sign determination and subsequent electron suppression. The design study presented here is based on a full simulation of the behavior of the experiment using the Geant 4 package[[]. In addition, key elements have been verified in a series of testbeams at CERN over the years 2006-2008.

PEBS (fig. 1 1 (left)) has been designed to meet the requirements stated above. A magnetic
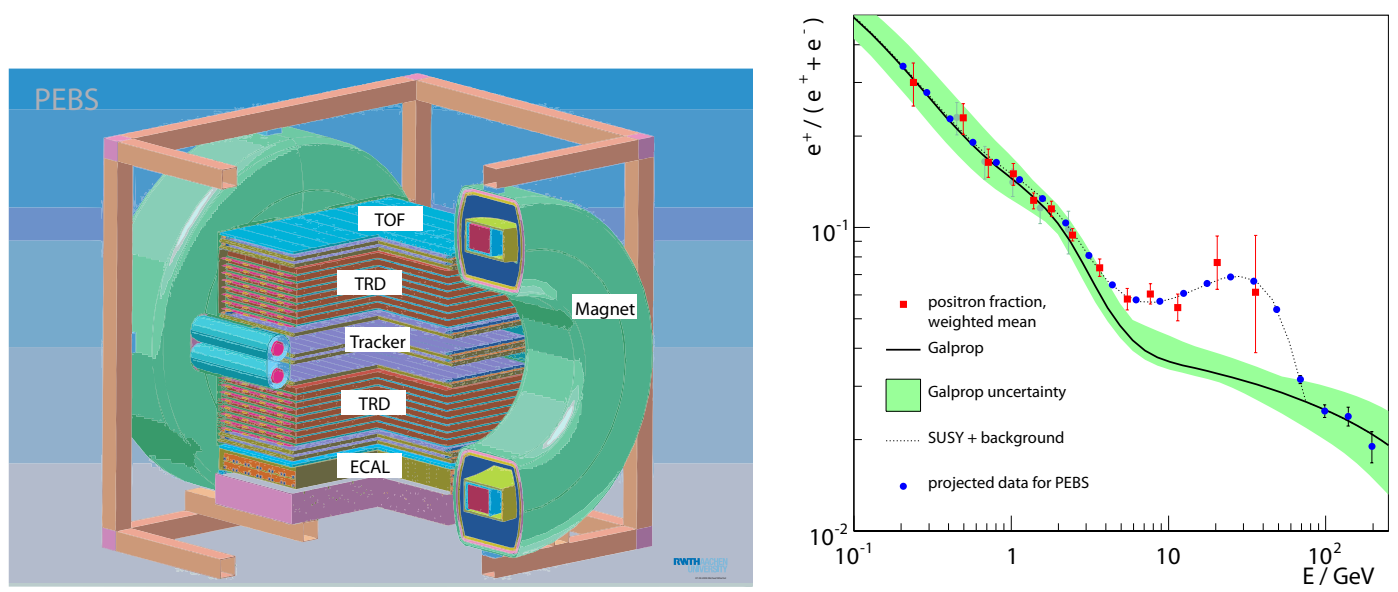

Figure 1: Left: Cut-out mechanical drawing of the PEBS detector. Right: Weighted mean of the positron fraction data from AMS-01[5], 6], HEAT[7], CAPRICE[8], and TS93[9], together with the secondary background as predicted by the Galprop[10] conventional model. The uncertainty band for the Galprop model was obtained from a variation of model parameters within the bounds allowed by the $\mathrm{B} / \mathrm{C}$ data and the positron fraction data below $3 \mathrm{GeV}$. The statistical uncertainties achievable with a detector that has a geometric acceptance of $0.4 \mathrm{~m}^{2} \mathrm{sr}$ and measures for 100 days are projected for the positron and electron fluxes of a dark matter scenario with an mSUGRA neutralino $\left(m_{0}=1560 \mathrm{GeV}, m_{1 / 2}=260 \mathrm{GeV}, \tan \beta=40, A_{0}=0\right.$ and $\operatorname{sgn} \mu=+1$ ) on top of the secondary backgrounds. A boost factor of 150 is used for the signal fluxes, chosen to give the best fit to the presently available data. DarkSUSY4.1[11] has been used for the calculation of the signal fluxes.

field of mean flux density $B=0.8 \mathrm{~T}$ and mean $B L^{2}=0.62 \mathrm{Tm}^{2}$ is created by two superconducting Helmholtz coils, located inside a helium cryostat. The curvature of a charged particle's trajectory in this field is measured by a scintillating fiber tracker with silicon photomultiplier readout[12]. A transition radiation detector (TRD), located between the tracker super-layers, and an electromagnetic calorimeter (ECAL) at the bottom of the experiment provide rejection power against protons. Scintillator panels above and below the tracker act as a time-of-flight system (TOF) and are used for triggering purposes. 
Earth's atmosphere prohibits a measurement of GeV-range cosmic rays on the ground. While space experiments have the undisputed virtue of being able to measure the spectra of cosmic rays completely undisturbed by the atmosphere, scientific high-altitude balloons constitute an interesting alternative for several reasons. The experiment can be salvaged after the flight and be recalibrated, refitted and eventually repeated for the gradual improvement of the statistical accuracy, and it can be conducted at a much lower cost. Mission durations of up to 40 days have been reached by traveling with the circular arctic winds around the North or South Pole[2]. The amount of helium carried for cooling the superconducting magnet will be sufficient for such a measurement time.

The geometric acceptance of the detector is limited by the weight and power constraints imposed by the carrier system. The most important contributions to the overall weight are the magnet weight and the weight of the calorimeter with $850 \mathrm{~kg}$ and $600 \mathrm{~kg}$, respectively. The power consumption is dominated by the $300 \mathrm{~W}$ needed for the tracker which has roughly 55000 individual readout channels.

The tracker has an aperture of $86 \times 86 \mathrm{~cm}^{2}$ and a length of $80.6 \mathrm{~cm}$. From the positions and dimensions of all the sensitive areas of the subdetectors, the geometric acceptance of PEBS is calculated to be $0.4 \mathrm{~m}^{2} \mathrm{sr}$.

The tracking device will consist of scintillating fibers grouped into modules and read out by linear silicon photomultiplier arrays (SiPMs). A module comprises two stacks of round fibers of $250 \mu \mathrm{m}$ diameter, 128 fibers wide and five fibers high, glued together in the tightest arrangement. The stacks are held apart by two carbon fiber skins with Rohacell foam in between. Using scintillating fibers, the material budget in the particles' flight path through the tracker does not exceed $6 \%$ of a radiation length, while the TRD will contribute another $6 \%$. The modules will be grouped into eight layers, two of those being located at the entrance and exit of the tracking device, respectively, and four in the center.

Silicon photomultipliers[3] have the virtues of being insensitive to magnetic fields, having high quantum efficiency, as well as compactness and auto-calibration. They will therefore be used to detect the photons trapped in the scintillating fibers and will be read out by a dedicated VA chip. Linear arrays containing 32 silicon photomultiplier columns each are located at alternating ends of the fiber bundles. The remaining end of each fiber is covered by a reflective coating to increase the light yield by a factor of roughly 1.6. Five fibers in one column are then optically connected to one SiPM column. The weighted cluster mean from amplitudes in adjacent SiPMs columns will be calculated to pinpoint the intersection of a trajectory with a fiber module.

A prototype of a tracker module has been subjected to a $10 \mathrm{GeV}$ proton testbeam at the CERN T9 beamline. The measured light yield of 11 photo electrons per MIP crossing was used as input to the PEBS Monte Carlo simulation. A momentum resolution of $16 \%$ is predicted for $100 \mathrm{GeV}$ protons in this case.

A sandwich calorimeter for three-dimensional shower reconstruction has been designed to provide rejection power against the predominant proton component in the cosmic rays. It comprises 20 layers consisting of $2 \mathrm{~mm}$ tungsten interleaved with layers of scintillator bars of $2 \mathrm{~mm}$ height and $7.75 \mathrm{~mm}$ width. They are read out by individual SiPMs of $1 \times 1 \mathrm{~mm}^{2}$ area which are sitting in front of wavelength-shifting fibers embedded in the scintillator bars on both ends. Attenuation is used on 
one side to increase the dynamic range. Five layers are grouped into a super-layer and four superlayers are placed with alternating direction. The total depth of the calorimeter is 11.4 radiation lengths.

A preliminary cut-based analysis, using the PEBS Geant4 simulation, has been performed to study the proton rejection of this setup. For each event, a shower fit using a standard Gamma function parameterization has been performed and the following variables have been used to distinguish positrons from protons: $E / p$-match, total shower amplitude, fitted shower maximum, ratio of shower energy within one Molière radius from the shower axis and angle between the reconstructed track and shower axis.

Proton rejections of the order of 1000 can easily be achieved already with this rather coarse method. The corresponding electron efficiency is around $75 \%$.

The design of the transition radiation detector is based on the one constructed for the AMS-02 experiment on the International Space Station[ [4. The TR x-ray photons are generated in a $2 \mathrm{~cm}$ thick irregular fleece radiator made of polyethylene and polypropylene. They are subsequently detected in proportional wire chambers in the form of straw tubes made of aluminized kapton foils which have an inner diameter of $6 \mathrm{~mm}$ and are filled with an $80: 20$ mixture of $\mathrm{Xe} / \mathrm{CO}_{2}$. The straw tubes are grouped into modules and eight layers each are placed in the gaps above and below the central tracking layers. The proton rejection yielded by the TRD reaches a value of 1000 at $80 \%$ electron efficiency in the interesting energy range.

\section{Performance study}

Detailed performance studies using both Monte Carlo and testbeam data have been conducted. As the ECAL and the TRD measure independently, their combined proton rejection power can be expected to be as high as one in one million. The large acceptance, good momentum resolution and reliable proton suppression of PEBS would allow a precision measurement of the cosmic-ray positron fraction (fig. 1 (right)) up to energies above $100 \mathrm{GeV}$.

As an example for the physics performance to be expected from PEBS, a scan of the mSUGRA parameter space was conducted. For the case that mSUGRA is realized in nature and the neutralino contained in this model constitutes the dark matter, the signal fluxes $\Phi^{\operatorname{sig}}\left(e^{ \pm}\right)$for positrons and electrons resulting from neutralino annihilations in the Galactic halo were calculated using DarkSUSY 4.1[11]. The model fluxes were calculated as the sum of the background fluxes $\Phi^{\mathrm{bg}}\left(e^{ \pm}\right)$, taken from the conventional Galprop model[13], and the boosted signal fluxes:

$$
\Phi^{\text {model }}\left(e^{ \pm}\right)=\Phi^{\mathrm{bg}}\left(e^{ \pm}\right)+f_{b} \cdot \Phi^{\mathrm{sig}}\left(e^{ \pm}\right)
$$

The boost factor $f_{b}$ in equation (2.1) was used as the only free parameter in a fit of the resulting positron fraction for a given point in mSUGRA parameter space to the weighted mean of the currently available data (fig. (1). The resulting contour of the best-fit $\chi^{2}$ is essentially flat over vast amounts of the parameter space (fig. 2 left). This situation can be expected to change drastically with the arrival of data from PEBS. For the benchmark model given in the caption of fig. 1, random data for an experiment with the acceptance of PEBS and a measurement time of 100 days were 

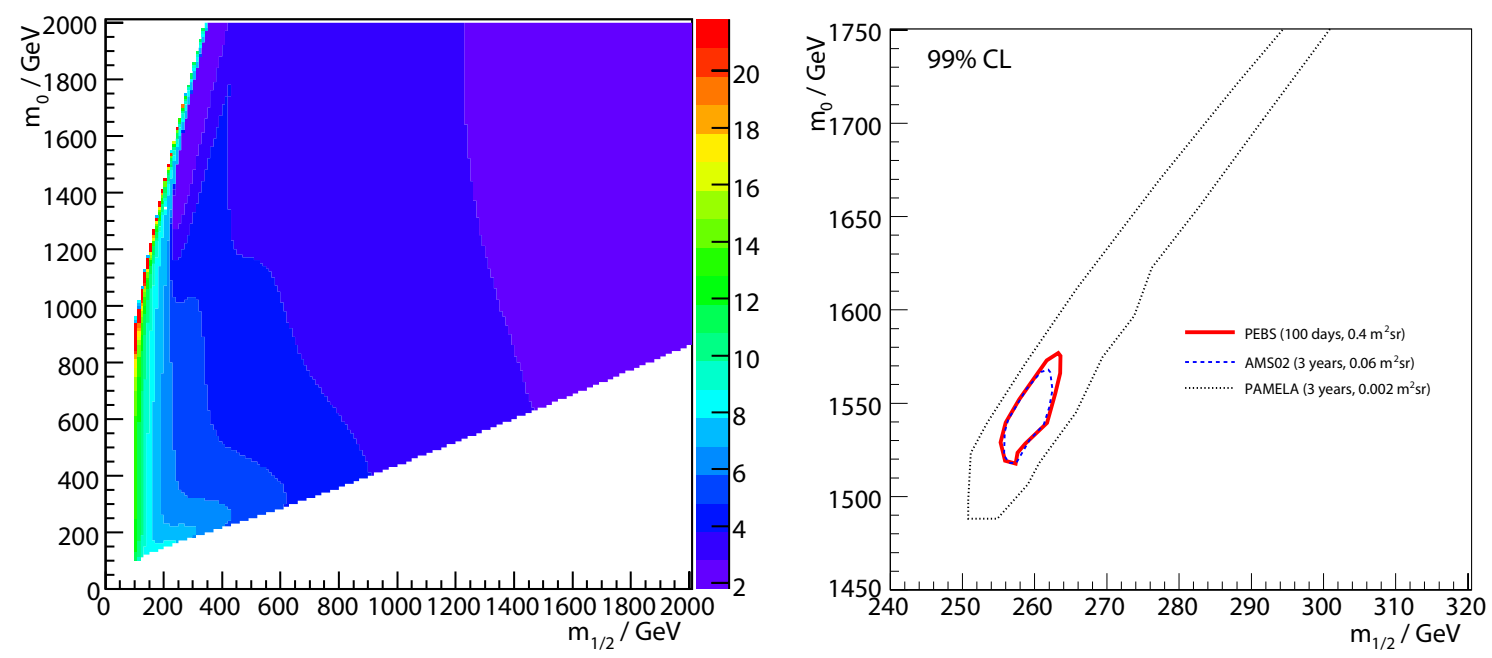

Figure 2: Left: $\chi^{2}$-contour as described in the text, for the weighted mean of the currently available positron fraction data. Right: Projected 99\% CL-contour for the statistical accuracy to be expected from PEBS, as compared to PAMELA and AMS-02, for the benchmark point used in fig. 1 (right), in the $m_{1 / 2}-m_{0}$-plane, for fixed $\tan \beta=40$. Note the different scales.

generated and the procedure described above was repeated. Now, the $\chi^{2}$-contour (fig. 2 right) has a distinct minimum at the benchmark point. In this model and at this level of statistical accuracy, the corresponding resolution of the neutralino mass would be limited by the energy resolution of the calorimeter which is expected to be on the order of $7 \%$ at $100 \mathrm{GeV}$.

\section{References}

[1] S. Agostinelli et al., Nucl. Instr. Meth. A 506 (2003) 250-303

[2] H.S. Ahn et al., Nucl. Instr. Meth. A 579 (2007) 1034-1053

[3] B. Dolgoshein et al., Nucl. Instr. Meth. A 563 (2006), 368-376

[4] P. v. Doetinchem et al., Nucl. Instr. Meth. A 558 (2006) 526-535

[5] J. Alcaraz et al., Phys. Lett. B 484 (2000) 10-22

[6] M. Aguilar et al., Phys. Lett. B 646 (2007) 145-154

[7] J.J. Beatty et al., Phys. Rev. Lett. 93 (2004) 241102

[8] M. Boezio et al., ApJ 532 (2000) 653-669

[9] R.L. Golden et al., ApJ 457 (1996) L103-L106

[10] A.W. Strong et al., Annu. Rev. Nucl. Part. Sci. 57 (2007) 285-327

[11] P. Gondolo et al., JCAP 0407 (2004) 008

[12] H. Gast et al., Nucl. Instr. Meth. A 581 (2007) 423-426

[13] V.S. Ptuskin et al., ApJ 642 (2006) 902-916 